\title{
A Focal Adhesion Filament Cross-correlation Kit for fast, automated segmentation and correlation of focal adhesions and actin stress fibers in cells.
}

\author{
Lara Hauke $^{1 \bullet}$, Shwetha Narasimhan ${ }^{2 \odot}$, Andreas Primeßnig ${ }^{1}$, Irina Kaverina ${ }^{2, \ddagger}$, Florian \\ Rehfeldt $t^{1,3} \ddagger$ \\ 1 Third Institute of Physics - Biophysics, Georg-August-University Göttingen, D-37077 \\ Göttingen, Germany \\ 2 Department of Cell and Developmental Biology, Vanderbilt University, Nashville, TN, \\ USA \\ 3 Experimental Physics I, University of Bayreuth, D-95440 Bayreuth, Germany \\ (2These authors contributed equally to this work. \\ $\ddagger$ Corresponding authors. \\ irina.kaverina@vanderbilt.edu; florian.rehfeldt@uni-bayreuth.de
}

\begin{abstract}
Focal adhesions (FAs) and associated actin stress fibers (SFs) form a complex mechanical system that mediates bidirectional interactions between cells and their environment. This linked network is essential for mechanosensing, force production and force transduction, thus directly governing cellular processes like polarization, migration and extracellular matrix remodeling. We introduce a tool for fast and robust coupled analysis of both FAs and SFs named the Focal Adhesion Filament Cross-correlation Kit (FAFCK). Our software can detect and record location, axes lengths, area, orientation, and aspect ratio of focal adhesion structures as well as the location, length, width and orientation of actin stress fibers. This enables users to automate analysis of the correlation of FAs and SFs and study the stress fiber system in a higher degree, pivotal to accurately evaluate transmission of mechanocellular forces between a cell and its surroundings. The FAFCK is particularly suited for unbiased and systematic quantitative analysis of FAs and SFs necessary for novel approaches of traction force microscopy that uses the additional data from the cellular side to calculate the stress distribution in the substrate. For validation and comparison with other tools, we provide datasets of cells of varying quality that are labelled by a human expert. Datasets and FAFCK are freely available as open source under the GNU General Public License.
\end{abstract}

\section{Author summary}

Our novel Focal Adhesion Filament Cross-correlation Kit (FAFCK) allows for fast, reliable, unbiased, and systematic detection of focal adhesions and actin stress fibers in cells and their mutual correlation. Detailed analysis of these structures which are both key elements in mechano-sensing and force transduction will help tremendously to improve quantitative analysis of mechanocellular experiments, key to understanding the complex interplay between cells and the extracellular matrix. In particular, sophisticated analysis methods such as model-based traction force microscopy will benefit from correlating the detailed datasets of stress fibers and focal adhesions. 


\section{Introduction}

The shape and mechanics of biological cells depends largely on the cytoskeleton, a dynamic network that functions as the cellular endoskeleton and produces contractile forces acting on their environment, such as the extracellular matrix (ECM) or neighboring cells. A predominant and essential part of this network is made up of actin filaments that act as structural elements and, importantly, are capable of producing contractile forces when co-assembled with myosin II mini-filaments into contractile stress fibers [1].

Geometry and rearrangement of stress fibers is a critical factor during mechanical interactions between the cell and the ECM in many processes (e.g. adhesion, migration, etc.) and must be quantitatively assessed to elucidate the complex mechanical interplay of cells with their surroundings. Interestingly, the pattern of stress fiber formation in human mesenchymal stem cells reveals an optimal matrix elasticity $E$ yielding an anisotropic and polarized acto-myosin fiber structure, which functions as an early morphological marker of mechano-guided differentiation [2,3]. This requires a quantitative analysis of the filament structure by an order parameter $S$, that builds on the unbiased and automated segmentation of stress fibers. Various approaches exist to address this task, among which our recently developed Filament Sensor analysis tool allows for automated detection and quantification of stress fiber structures [4]. However, for a complete functional analysis of cell and matrix mechanics, quantification of both stress fibers and their associated focal adhesions is needed.

Cells adhere to the ECM or surrounding cells via cell-matrix and cell-cell contacts, respectively. These structures function as biochemical anchors and are key to the signaling and mechanical interactions of cells with their surroundings. Focal Adhesions (FAs) are cell-matrix anchors based on trans-membrane proteins integrins, with a multitude of associated proteins on the cytosolic side. Serving as the interface between the SFs and the ECM, FAs have several functions, such as providing cellular attachment to the substrate, transducing contractile forces to the ECM and facilitating bi-directional transmembrane signaling [5]. At the cytosolic side, FAs are structurally and dynamically linked to the ends of SFs (see Fig 1A-C). The formation and maturation of FAs is dependent on actomyosin-generated tensile forces applied on them through associated SFs [5]. In turn, signaling pathways that are mechanically triggered at adhesions lead to actin polymerization and elongation of the fibers at their FA-associated termini [6]. Thus, there is an intricate, dynamic association between FAs and SFs that needs to be quantified to fully elucidate their cellular functionality.

Fig 1. Stress fibers and focal adhesions. Confocal fluorescence microscopy images of an MRC5 cell stained for $\mathbf{A}$ ) actin filaments (phalloidin) and $\mathbf{B}$ ) focal adhesions (paxillin). C) Merged color image of the cell with actin filaments in magenta and adhesions in green. All images are of the ventral plane of the cell, scale bar - $10 \mu \mathrm{m}$. D) Schematic illustration of different stress fiber subtypes and their association with focal adhesions.

Cellular SFs are broadly classified as transverse arcs, dorsal SFs and ventral SFs based on their FA association, which underlies their varied roles (Figure 1D) [1]. Actin transverse arcs, which are not associated with FA but rather embedded into the cortical actin meshwork at their termini, are contractile structures that contribute to cell shape but not do not directly exert force onto the environment. Dorsal SFs are associated with FAs at one end and with transverse arcs on the other end. Although they are non-contractile due to their negligible myosin II content, they can exert forces on their terminal adhesion through their association with transverse arcs. Ventral SFs, which are 
connected to FAs at both ends, are contractile structures that generate majority of cellular traction forces on the substrate $[7,8]$. Due to this natural linkage of SFs and FAs, cytoskeletal studies often result in cells with an observed actin SF phenotype having an associated FA phenotype [9-13]. Therefore, incorporating detection of SF-FA coupling in studies would greatly facilitate the complete analysis of their structure and function in cells.

Manual evaluation and analysis of FAs and SFs is a laborious, time-intensive process and is always at risk due to the observer's bias. Recently, this process has been aided by several automated analysis tools and algorithms that are optimized either for focal adhesion analysis (such as the Focal Adhesion Analysis Server [14] and other methods $[15,16]$ ), or stress fiber analysis (such as previous version of Filament Sensor [4], CytoSeg [17] and other tools [18,19]).

However, a tool for speedy, unbiased quantification of SFs, FAs, and their mutual coupling is yet missing. Here, we present an integrated FA-SF analysis module called the Focal Adhesion Filament Cross-correlation Kit (FAFCK). This tool is based on our previously published Filament Sensor analysis tool, with added capacities for adhesion detection and characterization, filament analysis and coupled FA-SF correlation for stacks or pseudo-stacks of images with similar properties to streamline analysis of huge datasets. FAFCK detects and quantifies FAs and SFs by means of location, area, length, width, aspect ratio and orientation, with capacity for exporting this information enumerated for each frame, allowing for comprehensive further data analysis (e.g. Python, Matlab, etc.) to elucidate cell and matrix mechanics. Our software package will be particularly helpful for sophisticated mechanical measurements and analysis such as model based traction force microscopy (MBTFM) experiments [7] that takes advantage of the a priori determined positions of focal adhesions and stress fibers in addition to the displacement field in the substrate.

\section{Results}

The Focal Adhesion Filament Cross-correlation Kit (FAFCK) is a comprehensive FA-SF analysis software consisting of two modules: the FASensor, for adhesion detection and the Filament Sensor, for actin filament detection, both of which connect through a correlation function for paired characterization of these structures. To correlate an adhesion with the associated actin filament in the cell, the software relates each adhesion object detected by FASensor with corresponding filaments that are detected by the Filament Sensor module. As stand-alone programs with a shared GUI, both routines can be used independently as well.

\section{Segmentation of focal adhesions by FASensor}

The FASensor is the adhesion detection module in the software. It is a robust tool for detection of point-like structures partly based on the Filament Sensor [4]. Based on adapted ImageJ routines (Fig 2), it analyzes the adhesions in an image as objects which can be exported with characteristics and IDs, with multiple customization options to improve accuracy as desired by the user.

Fig 2. Workflow of adhesion detection by FASensor.

Adhesion detection analyzes and segments the input image (usually a grayscale immunofluorescence (IF) micrograph) of focal adhesions. The module is split into Main, Pre-processing and Focal Adhesion output sections. All images are shown in panels on 
the right- including the original image of adhesions, the pre-processed image, the thresholded image, and the image with overlay of filaments detected from the filament input (Stress Fiber Overlay). The windows can be split from the interface and zoomed in for user ease. The pre-processing tab allows the user to add optional filters to the image in order to improve the signal to noise ratio and normalize the image. Filters included are the Gauss filter, Laplace filter, Line Gauss filter, Cross-correlation filter, and Enhance contrast filter. Filter queues can be saved for reuse. The main tab has thresholding controls with automated protocols. The levels can also be altered manually to produce the desired binarized image. Additional filters are provided for defining the minimum or maximum pixel number per adhesion and the maximum amount of clusters allowed in one image.

On clicking 'Process Focal Adhesions', the adhesion objects are detected. For each adhesion detected, the outline is derived, and a convex hull is calculated. The main axis is set for the points farthest away on the convex hull and for the points farthest away from the main axis, the side axis is set. The aspect ratio, orientation, and center for each focal adhesion is also calculated. The module also allows for further close customization of the detected objects by the user to obtain the most accurate result. In cases where nearby adhesions have been detected as a single one due to poor signal-to-noise ratio, overlap, artifacts, etc. the user has the option to draw a line on the thresholded image and separate the adhesions at their discretion (see Fig 3C). Once the lines have been drawn to separate all adhesions as desired, the adhesions can be re-processed to get the split objects in a new map.

The detected FAs are displayed in the table in the Focal Adhesion tab. The ID, XY center position, Length of main axis, Length of side axis, Angle, Area, and Area ellipse of each adhesion are listed in the table. The user can choose to discard a detected adhesion object by selecting the object in the Focal Adhesion original window, on which the boundary turns red, and clicking the remove button under the table. This allows the user to closely edit the adhesion map obtained from the software to remove any inconsistencies based on their expertise. The output focal adhesion map can be exported as a binary mask with outlines and optional numbering with IDs. The output table can be exported as a 'CSV' file and the adhesion detection can be exported as a project 'XML' file.

We illustrate the usage of FASensor with the input image of an MRC5 human fibroblast cell showing adhesions (paxillin) (see Fig 3A). This input file was preprocessed using Laplace and Gauss filters and thesholded using Intermodes algorithm. With a minimum limit of 10 pixels per adhesion, which corresponds to $0.144 \mathrm{\mu m}^{2}$, adhesion objects were detected by the software (Fig 3B). 
Fig 3. Segmentation of FAs by FASensor and subsequent optimization. A) Input image of focal adhesions (paxillin) in the ventral plane of an MRC5 cell. B) Corresponding segmented adhesion objects (outlined in green) from FASensor. C) Montage of adhesion splitting capacity of the FASensor module. (Top) Thresholded image of adhesions (white) have objects detected by module (circled by yellow). Red line is drawn by user to split objects where desired. (Bottom) Before and after images of objects detected in the IF adhesion input, that are split. Green arrow indicates splitting objects in Top and Bottom. D) Montage showing the closing and filling holes function of the module. (Top series) Objects circled by green detected by FASensor from IF adhesion input. (Bottom series) Objects circled by yellow on thresholded image. (Left) Pre-detection by module (Center) Objects detected when closing and filling holes algorithms are not applied. (Right) Objects detected when closing and filling holes algorithms are applied. Green arrow in the Top and Bottom series indicates the adhesion which is detected as multiple objects without the algorithm and detected as a single object with the algorithm.

In the post-thresholding section, there is the option to add or opt-out of the closing and filling holes algorithms, by which seemingly disparate objects can be detected as one, especially in cases of large, single adhesion plaques whose signal is not uniform (Fig 3D). A large, boundary adhesion plaque that is detected as split pieces without the closing and fill holes algorithm can be re-processed with this algorithm in order to assign it as a single object, which is accurate with user's expert perception of the IF image.

\section{Evaluation of the FASensor output with user generated output}

For those using this module to analyze cellular adhesions, it is important to understand how the results compare to their expert opinion and any pre-established routines they already use. To accurately assess the differences between the user expert's routine and the FASensor output in adhesion detection, our module offers an evaluation option.

In the evaluation panel, a binary adhesion map generated by the user can be compared with the adhesion object output generated by FASensor software from the IF image (Suppl Fig 1A). Before comparison, additional pre-processing can be applied, for example thickening of outlines. The comparison is done in two ways - an objectwise fashion, where from both the user mask and software output, objects are generated and overlap is checked, and in a pixelwise fashion, where each pixel of user mask and software output is taken into account. The minimal required overlap for object matching between the user mask and output can be manually set by the user. The 'export results' option provides images of the comparisons and comparison results in a csv file. The results table lists objects that are found in output when compared with user mask, objects that are false positives (present only in the software output image, labelled 'eval not matched') and the missed objects that are present only in the user's image (labelled 'truth not matched'). The pixel sizes of all objects are given along with the number of pixels that overlap in the common objects. The output table also gives the cases where the sensor detects multiple objects in output for one object in the mask marked by the expert (multiMatchesOneToN) and cases where the sensor detects one object in the output for multiple objects marked in the mask by the expert (multiMatchesNToOne).

In the example, the binary mask (Suppl Fig 1B) has been generated by a human expert marking the adhesions from the IF image using Fiji software [20]. On the landing page of the graphic user interface, the user can also import two binary masks to execute evaluation without running the software first. As all parts of the software, the evaluation tool can work on OME-TIFF files to provide fast evaluation of large datasets. 
Suppl Fig 1C shows the result maps of objectwise and pixelwise evaluation between FASensor output and the user mask. The evaluation maps are based on the user mask, highlighting the found and missing categories on it and superimposing false positives from output on the mask as well. The tables Suppl Fig 1D and E show the tabulated results for the different categories in objectwise and pixelwise evaluation respectively.

The input routine, in terms of filters and thresholding method used, affects similarity of the FASensor output to user mask. Suppl Fig 2 shows how objects detected by FASensor are more similar to the user mask when an appropriate input routine is used. From input of (Suppl Fig 2A), FASensor output is derived in two ways: unoptimized (filter settings and thresholding that is not appropriate for the cell) and optimized (appropriate filter settings and thresholding). Objectwise evaluation of the outputs with user mask (Suppl Fig 2B) is shown in Suppl Fig 2C for unoptimized output andSuppl Fig 2D for optimized output, where found and missing categories as compared to output on the left are highlighted on the user mask and false positives from output are superimposed on the mask as well. In the unoptimized output, the pronounced background signal at the input cell border is fused as large plaques, detected objects deviate from the user mask and many false positives are present. By using an appropriate, optimized input routine, the focal adhesion signal is separated well from the background and adhesions are detected. More detected adhesions match with the user mask and false positives are largely diminished as well. The results are summarized in Suppl Fig 2E. There is an increase in the multiMatchesOneToN parameter for the optimized routine, because the optimized input routine finely detects adhesions in the boundary areas of high background, where some of them have been marked as large single adhesions by the user when the signal couldn't be distinguished finely by eye. Thus, several objects detected by the output in these areas are matched to one object marked by the user.

Conversely, if the output had detected a large object from signal that was distinguished as several objects by the user, that would result in an increase in the multiMatchesNToOne parameter.

\section{FASensor output performance with varying imaging conditions and levels of optimization}

To test FASensor's robust detection of focal adhesions on a variety of image qualities, we compiled comparison datasets with varying degrees of blur, in which structures were manually labelled by a human expert for comparison. MRC5 cells immunostained for actin filaments and focal adhesions were imaged on a confocal laser-scanning microscope (Suppl Fig 3) in three conditions with blur introduced in images by altering the size of pinhole to include out-of-focus light. For the Confocal in-focus dataset (Suppl Fig 3A), the pinhole size was 1.2 Airy Units (AU), for the Confocal mild blur dataset (Suppl Fig 3B), the pinhole size was $3 \mathrm{AU}$ and for the Confocal severe blur dataset (Suppl Fig 3C), the pinhole size was $4.7 \mathrm{AU}$.

Since the Filament Sensor module has been analyzed and published before, we have focused on the FASensor module for manual annotation comparison. We analyzed a set of adhesion images from each imaging condition (in-focus, mild blur and severe blur) in the FASensor software and compared the software results with adhesions manually annotated for the respective images. For manual annotation by the user expert, selected images from the sets were marked for adhesions using the freehand selection tool in Fiji with the aim of being natively user-detected. Images were traced with minimal signal manipulation to compare the base-level manual annotation by eye with the objects traced by FASensor module after processing by software.

To further understand whether and how user involvement such as pre-processing 
each image in a set differently or splitting ROIs and excluding adhesions makes a significant improvement in software results, we used three different optimization levels. In the unoptimized (UN) level, the user sets a single desired input routine with thresholding and pre-processing parameters for all images in the dataset and derives results from the software. There is no optimization for each cell in the dataset and user involvement is low. In the optimized (OP) level, the user sets a custom input routine for each cell with the optimal thresholding and pre-processing parameters and derives results from the software. This optimization uses the software's capability for pre-processing and thresholding to enhance adhesion recognition for every cell according to user's discretion. The user involvement is greater than unoptimized in that every cell has a different optimal setting. In the customized (CM) level, the user sets a custom input routine for each cell and further edits the result by splitting ROIs and deleting adhesions detected so that the result is highly customized and similar to the user manually marking the adhesions. Customization is useful for conditions where the user does not have the time to mark adhesions manually but still desires the detected adhesions to exactly fit to their discretion of the adhesion pattern in an image. The user involvement is thus higher than unoptimized and optimized levels.

Comparison of the software output with the manually marked adhesions gives result categories of adhesions that are found, missed or false positives. To compare these three results in the three optimization conditions, we created a similarity coefficient $(\mathcal{S C})$ for adhesion detection that is as follows:

$$
\mathcal{S C}=\frac{\sum \text { Found FA area }}{\sum \text { Missed FA Area }+\sum \text { False positive FA area }}
$$

The higher the coefficient, the more similar the detected adhesions are to the human expert's mask.

For the cells in the In-Focus dataset (Fig 4A), the similarity coefficients show that adhesions detected in $\mathrm{OP}(\overline{\mathcal{S C}}=12.73)$ and $\mathrm{CM}(\overline{\mathcal{S C}}=14.83)$ sets are significantly more user-similar compared to the UN set $(\overline{\mathcal{S C}}=4.53)$. The similarity coefficient of the OP and $\mathrm{CM}$ sets are not significantly different. Just setting optimal pre-processing settings vastly improves similarity of detected adhesions between cells in the in-focus set, even without further time-intensive customization of splitting and deleting detected objects.

Fig 4. Similarity coefficient $(\mathcal{S C})$ for different levels image quality and optimization. $\mathcal{S C}$ on the y-axis (logarithmic scale) for unoptimized 'UN' (purple squares), optimized 'OP' (blue triangles) and for customized 'CM' (green hexagons) output of analyzed images of the $\mathbf{A}$ ) confocal in-focus set $(\mathrm{n}=17$, UN $\overline{\mathcal{S C}}=4.53$, OP $\overline{\mathcal{S C}}=12.73$ and $\mathrm{CM} \overline{\mathcal{S C}}=14.83), \mathbf{B})$ confocal mild-blur set $(\mathrm{n}=17, \mathrm{UN} \overline{\mathcal{S C}}=4.47$, OP $\overline{\mathcal{S C}}=7.98$ and $\mathrm{CM} \overline{\mathcal{S C}}=10.13)$ and $\mathbf{C})$ confocal severe-blur set $(\mathrm{n}=19$, UN $\overline{\mathcal{S C}}=3.47$, OP $\overline{\mathcal{S C}}=5.94$ and $\mathrm{CM} \overline{\mathcal{S C}}=11.74) .{ }^{* * *} \mathrm{p}<0.001 ;^{* *} \mathrm{p}<0.01{ }^{*} \mathrm{p}<0.05$ and ns stands for not significant $(\mathrm{p}>0.05)$.

For the cells in the mild-blur dataset (Fig 4B), the similarity coefficients show that OP set $(\overline{\mathcal{S C}}=7.98)$ is not significantly more similar than $\mathrm{UN}(\overline{\mathcal{S C}}=4.47)$ or $\mathrm{CM}$ $(\overline{\mathcal{S C}}=10.13)$, whereas CM is significantly more similar than UN. Thus, in conditions where images have some blur, doing both optimal pre-processing and user customization by splitting and deleting adhesions makes it significantly more accurate.

For cells in the severe-blur dataset (Fig $4 \mathrm{C}$ ), the similarity coefficients show that OP set $(\overline{\mathcal{S C}}=5.94)$ is not significantly more similar than $\mathrm{UN}(\overline{\mathcal{S C}}=3.47)$, but again $\mathrm{CM}$ 
$(\overline{\mathcal{S C}}=11.74)$ is significantly more similar than both other sets. Thus, in conditions where images are blurred, intensive user customization by splitting and deleting adhesions gives the best result.

Aggregate analysis (Suppl Fig 4) of all the adhesions in the sets reveal that false positive adhesions are consistently significantly smaller than found and missed adhesions across optimization levels and missed adhesions are significantly smaller than found adhesions as well. (Fig 5) shows graphs comparing the adhesion objects by area in a set across optimization levels.

Fig 5. Comparison of adhesion objects between optimization levels in a set. Graphs show pooled adhesion objects for un-optimized 'UN', optimized 'OP' and customized 'CM' analysis. Y axis has adhesion area in $\mu^{2}$ on a logarithmic scale. Left column shows graphs comparing adhesion objects found in common between user mask and software output. Middle column shows adhesion objects that were missed in output and present only in user mask. Right column shows adhesion objects that are false positive, present only in the software output. A) Graphs for in-focus set B) Graphs for mild-blur set C) Graphs for severe-blur set. ${ }^{* * * *} \mathrm{p}<0.0001 ;{ }^{* * *} \mathrm{p}<0.001 ;{ }^{* *} \mathrm{p}<0.01$; ${ }^{*} \mathrm{p}<0.05$ and $\mathrm{ns}$ stands for not significant $(\mathrm{p}>0.05)$.

Aggregate analysis of the in-focus set (Fig 5A) shows that there is no significant difference between area of the found objects across optimization levels, but both OP (1843) and CM (1802) find more adhesions compared to UN (1451). OP (268) and CM (309) miss less adhesions than UN (660) and CM missed adhesions are significantly smaller than UN. Optimization decreases false positive adhesion area significantly- with $\mathrm{CM}\left(\bar{A}=0.33 \mu \mathrm{m}^{2}\right)$ and $\mathrm{OP}\left(\bar{A}=0.25 \mathrm{\mu m}^{2}\right)$ adhesions being significantly smaller than UN $\left(\bar{A}=0.43 \mathrm{\mu m}^{2}\right)$ false positive adhesions. OP shows the largest decrease in false positive adhesion size, but CM (296) has the largest decrease in number over UN (497) and OP (465). Thus, optimization of in-focus images primarily increases the accuracy by finding more objects and decreasing false positives by number and area.

Aggregate analysis of the mild blur set (Fig 5B) shows that there is no significant difference between the area of found objects across optimization levels, but both OP (1740) and CM (1700) find more objects compared to UN (1522). In missed objects both OP $\left(\bar{A}=0.66 \mathrm{~mm}^{2}\right)$ and CM $\left(\bar{A}=0.64 \mu^{2}\right)$ have smaller missed adhesion area compared to UN $\left(\bar{A}=0.83 \mu \mathrm{m}^{2}\right)$, and less missed adhesions (OP (361), CM (401)) than UN (579) as well. Comparing the false positive objects, OP has lower $\bar{A}\left(\bar{A}=0.31 \mathrm{\mu m}^{2}\right)$ compared to UN $\left(\bar{A}=0.42 \mu^{2}\right)$ and $\operatorname{CM}\left(\bar{A}=0.43 \mu \mathrm{m}^{2}\right)$, the number of false positives (560) is lower than UN (791) but higher than CM (188). Thus, in mild blur images, optimization method shows improvement over unoptimized by finding more adhesions and having fewer false positives. Customization shows much fewer false positives compared to other levels.

Aggregate analysis of the severe blur set (Fig 5C) shows that there is no significant difference between area of found objects across optimization levels, but both OP (1520) and CM (1577) find more adhesions than UN (1504). In missed objects, both OP $(\bar{A}=$ $\left.0.61 \mathrm{\mu m}^{2}\right)$ and $\mathrm{CM}\left(\bar{A}=0.60 \mathrm{\mu m}^{2}\right)$ are significantly smaller than $\mathrm{UN}\left(\bar{A}=0.85 \mathrm{\mu m}^{2}\right)$. More adhesions are missed in UN (355) compared to OP (339) and CM (282) as well. Comparing the false positives, UN $\left(\bar{A}=0.43 \mu^{2}\right)$ has the highest mean area $\bar{A}$ compared to OP $\left(\bar{A}=0.36 \mu^{2}\right)$ and $\mathrm{CM}\left(\bar{A}=0.36 \mu^{2}\right)$. OP (745) and CM (231) have lower number of false positives compared to UN (1811) as well. Thus, for severely blurred images, optimization method shows improvement over unoptimized by finding more adhesions and having fewer false positives. Customization shows a greater improvement by having much fewer missed adhesions and false positives compared to other levels. 
Thus FASensor offers several levels of desired user optimization in a variety of imaging conditions to derive and evaluate an accurate adhesion map from the input.

\section{Detection of Stress Fibers with Filament Sensor}

The Filament Sensor, as integrated here in the FAFCK, is based on the version published by Eltzner et al in 2015 [4], adjusted to feature stack handling of image sequences and drastically reduced runtime as well as some additions for area calculation. The plugin featured in the FAFCK includes all components included in the stand-alone software, being a preprocessing, line sensor, and filament submenu. The workflow of the software as published before is included in Suppl Fig 5 .

During preprocessing, contrast and brightness can be adjusted for either individual pictures or a whole stack, if needed. These routines are based on ImageJ [21], which is included as an internal library and used wherever possible, as the ImageJ routines are fast and well tested. The main preprocessing step consists of a filter queue which the user can customize to their needs. This serves as the 'artificial retina' to prepare the original IF filament image for binarization, tackling the issue of crossing filaments that have to be recognized as individual structures. On this image, the binarization is applied and filament objects extracted according to the flowchart shown in Suppl Fig 5. This is done in parallel threads to improve runtime and subjected to several boundaries the user can determine including minimal and maximal length, maximal curvature, width, restriction to cell area mask, and more. This flexibility allows for the program to be utilized for a wide variety of filament types. Lastly, the filament subsection allows to filter data for export. The Filament Sensor module offers a set of descriptors of the whole cell such as IDs, area, aspect ratio, length of axes, number of filaments, orientation, brightness, and for each individual filament such as xy position, length, curvature, width, and orientation. For each image file, the filament objects are assigned an individual identifier as done for the focal adhesion objects and a variety of export types are available with the option of superimposing filaments as required.

\section{Correlation of detected focal adhesions and actin filaments in FAFCK}

As focal adhesions and actin filaments are linked structures, the FAFCK module offers correlation of detected focal adhesions from FASensor and filaments from Filament Sensor. The software's workflow is illustrated in Fig 6 .

\section{Fig 6. Workflow for correlation in FAFCK.}

Using the file name of the original images or image stacks loaded, it is first checked whether input data for both adhesions and filaments exist and single sets are ignored. The focal adhesion objects detected from the input image showing paxillin are paired with the filaments derived from the input image depicting actin (see Fig 7A, B).

Starting from the ends, for every point in a filament, every focal adhesion in the specified neighborhood is checked with the requirement that the focal adhesion main axis has to be longer than the filament length. For the focal adhesion objects, the user decides whether the convex hull or the fitted ellipse is used for verification purposes. This ellipse is calculated by setting the line between the two points with the greatest distance on the convex hull as long axis and the axis orthogonal to that and with the greatest length as short axis. Furthermore, the area can be artificially increased by increasing the neighborhood in which verification is done. Now, starting from the ends, for each point on the filament, a neighborhood rectangle is created and in the list of

287
288
289
290
291
292
293
294
295
296
297
298
299
300
301
302
303
304
305
306
307
308
309
310
311


Fig 7. Correlation of focal adhesions and actin filaments by FAFCK. A) Input images of the ventral plane of a MRC5 cell (left) adhesions (paxillin) and (right) actin filaments (phalloidin). B) Map of numbered adhesion objects detected by FASensor (left), map of filaments detected by Filament Sensor (right). C) Color coded map of correlated adhesions and filaments categorized by association (legend in image). D) Aggregate graph of lengths of filaments categorized by adhesion association in all cells of the in-focus dataset. Y axis is in logarithmic scale. MAAF- Multiple adhesion associated filament, SAAF - Single adhesion associated filament and NAAF - Not adhesion associated filament. E) Aggregate graph of areas of adhesions categorized by filament association in all cells of in-focus dataset. $\mathrm{Y}$ axis is in logarithmic scale. AAMF - Adhesion associated with multiple fibers, AASF - Adhesion associated with single fiber and AANF - Adhesion associated with no fibers.

focal adhesions with main axis length below filament length, intersecting objects are searched.

The correlation can be done with condition of either validating all filaments that are attached to at least a single adhesion or only validating those with multiple adhesion structures along the filament. Thus, we can clearly categorize filaments by the number of adhesions associated. The data of adhesions by number of filaments associated can also be derived.

The output of the correlation routine consists of the identifier numbers of the respective objects and can consecutively be matched to the data output of the previous routines. Also, verified filaments will be highlighted in the fiber overlay and after verification the fiber data export will be expanded by a 'verification' column with booleans. As with the individual modules themselves, batch analysis of correlation for pairs of FA and SF images are possible as well.

The output is displayed in the Stress Fiber Overlay window in the main tab in FAFCK. The resulting paired filament and focal adhesion IDs are displayed in a table in the Focal Adhesion tab. The results can be exported as a simple overlay or a comprehensive color-coded map (Fig 7C), verifier tables, and grouped CSV files with details of adhesions and filaments by association with each other.

We used the FAFCK to correlate detected adhesions with filaments in the in-focus dataset $(\mathrm{n}=17)$. We named filaments by adhesion association as MAAF- Multiple adhesion associated filament, SAAF - Single adhesion associated filament and NAAF Not adhesion associated filament. There were 490 MAAFs, 588 SAAFs and 555 NAAFs, with MAAFs having significantly higher mean fiber length of $13.14 \mathrm{\mu m}$ compared to SAAFs at $9.29 \mu \mathrm{m}$ and NAAFs at $7.15 \mu \mathrm{m}$ (Fig 7D). Thus, filaments attached to multiple adhesions are longer than those attached to only one adhesion or none. We also analyzed adhesions by number of fibers associated with them and grouped them as AAMF - Adhesion associated with multiple fibers, AASF - Adhesion associated with single fiber and AANF - Adhesion associated with no fibers. AAMFs were much fewer (291) than AASFs (1191) and AANFs (1227) and had significantly larger mean adhesion area $\left(\bar{A}=1.21 \mathrm{\mu m}^{2}\right)$ compared to AASFs $\left(\bar{A}=0.64 \mu^{2}\right)$ and AANFs $\left(\bar{A}=0.34 \mu^{2}\right)$ (Fig $7 \mathrm{E})$.

The correlation analysis provides a comprehensive picture of adhesion and filament association in cells, and can be used to streamline quantitative evaluation of the effective mechanical forces in the stress fiber / focal adhesion system. 


\section{Discussion}

Here we present the FAFCK that allows for fast, reliable, unbiased, and systematic detection of fibers and point-like structures and their cross-correlation in cells. While detection and analysis of both types of structures individually is useful, the cross-correlation module will be especially valuable and help to answer many burning questions on the coupled function of these force-transmitting features in cellular mechanosensing.

There are several notable advantages to our new tool. Importantly, it allows to identify groups of stress fibers associated with zero, one, or more than one focal adhesion. Such classification can be applied to functional differences of stress fibers in cells of specific morphologies. For example, in migrating cells, this allows for an quantification of the relative number and characteristics of transverse arcs (0 FA per filament), dorsal SFs ( 1 FA per filament), and ventral SFs ( $\geq 2$ FAs per filament) in large data sets. This analysis can also be applied to other types of actin organization in specialized cell types. This software functionality allows to recognize different types of actin bundles in 2-dimensional images, such as maximum intensity projections of confocal stacks, which significantly quickens the quantification (in comparison with the necessity to detect structures in three-dimensional space, for example, dorsal stress fibers ). Furthermore, individual application of filters and optimization allows for an optimal analysis of wide-field images and images with high blur and/or background noise.

There is always a certain degree of error or deviation in computational recognition methods (as false positives and false negatives) as well as bias in the user's native detection of cellular features. Our software package allows for the systematic, streamlined, and unbiased comparison of large data sets to achieve statistical relevance. We expect that FAFCK will be very useful for analysis of time lapse movies, where many frames need to be analyzed consecutively to quantify the dynamics of stress fibers and adhesions in cells to understand their dynamic organization and how they influence the mechanical coupling of cells and the matrix. Since we provide an option to customize output in each image, this also allows for more precise detection of SF types in smaller data subsets. While our original motivation for this project was the quantitative analysis of focal adhesion structures and their correlation with stress fibers, this tool can be also used for image analysis of other cellular structures from fluorescence microscopy images. This includes but is not limited to membrane organelles such as lysosomes or mitochondria, that can be detected and also tracked to quantify their cellular dynamics.

For optimal flexibility and potential comparative studies, we provide an import option of external data sets of filaments and FAs (source may be manual detection or from other software). This feature allows for comparison of the computational recognition with individual user perception of the biological reality and also allows for importing data from other image analysis platforms to be used for the correlation analysis. In the light of the continuous improvement of image recognition software in the field we specifically refrained from employing machine learning and big data algorithms to establish a solid classical analysis tool. That said, the future development of FAFCK can surely benefit from big data and deep learning additions.

\section{Author Contributions}

L.H. designed and programmed the software, tested the software and co-wrote the manuscript. S.N. performed cellular experiments and imaging, analyzed the data, tested the software and co-wrote the manuscript. A.P. programmed the software under guidance of L.H. and F.R.. I.K. and F.R. designed and directed the project and edited the manuscript. All authors provided intellectual input in development of the project
365

366

367

368

369

370

371

372

373

374

375

376

377 378 380 381 382 383 384 385 379 
and vetted the final manuscript.

\section{Acknowledgements}

414

We thank Hamida Ahmed for technical assistance. This publication was funded by the German Research Foundation (DFG) and the University of Bayreuth in the funding programme Open Access Publishing.

\section{Funding}

This work was supported by the National Institutes of Health (NIH) grant R35-GM127098 to I.K. and R01-DK106228 to I.K.. S.N. was supported by an American Heart Association (AHA) pre-doctoral fellowship (18PRE33990479). F.R. and L.H. greatly appreciate funding by the Deutsche Forschungsgemeinschaft (DFG) via the Collaborative Research Centers (SFB) 755 (project B8) and 937 (project A13). This publication was funded by the German Research Foundation (DFG) and the University of Bayreuth in the funding programme Open Access Publishing.

\section{Conflicts of Interest}

The authors declare no conflict of interest.

\section{Materials and methods}

\section{Cell culture}

MRC5 cells (human lung fibroblasts, ATCC ${ }^{\circledR}$ Cat\# CCL-171 ${ }^{\mathrm{TM}}$, RRID:CVCL_0440) were maintained in MEM media (Cat\# 11095080, Thermo Fisher Scientific) supplemented with $10 \%$ fetal bovine serum, $100 \mu \mathrm{M}$ penicillin and $0.1 \mathrm{mg} / \mathrm{ml}$ streptomycin in $5 \% \mathrm{CO}_{2}$ at $37^{\circ} \mathrm{C}$. Media was supplemented with $5 \mu \mathrm{g} / \mathrm{ml}$ Plasmocin (Cat\# ant-mpp, InvivoGen) as a prophylactic against mycoplasma contamination.

\section{Fixation and immunostaining}

MRC5 cells were seeded on glass coverslips (Cat\# NC1129240, Fisher Scientific) that had been coated with $10 \mathrm{\mu g} / \mathrm{ml}$ fibronectin (Cat\# FC010, EMD Millipore) for 1 hour. After 24 hours, cells were fixed with $4 \%$ paraformaldehyde prepared in CB (cytoskeletal buffer - $150 \mathrm{mM} \mathrm{NaCl}, 5 \mathrm{mM} \mathrm{MgCl} 2,5 \mathrm{mM}$ EGTA, 5mM glucose, 10mM MES), for 10 minutes at room temperature. They were washed with $\mathrm{CB}$ after fixation, permeabilized with $0.25 \%$ Triton in CB. Antibodies used are as follows: anti-paxillin mouse primary antibody (1:200, BD Biosciences Cat \# 610051, RRID:AB_397463), Alexa Fluor 568 Phalloidin (1:300, Invitrogen, Cat\# A12380) and Alexa Fluor 488 conjugated goat anti-mouse IgG secondary antibody (1:300, Thermo Fisher Scientific Cat\# A-11001, RRID:AB_2534069). Coverslips were post-fixed for 10 min with $4 \%$ PFA in CB at room temperature. They were mounted with Vectashield Mounting Medium (Cat \# H-1000-10, Vector Labs) on glass slides (Cat \# 12-550-343, Fisher Scientific).

\section{Confocal microscopy}

Immunostained samples were imaged using a laser scanning confocal microscope- Nikon A1R HD25 configured with a Ti2-E inverted microscope, with a $100 \times$ oil immersion 
objective (MRD01991, N.A.=1.49). Three different pinhole settings were used to adjust the amount of out-of-focus light in the images- 1.2 AU (small, in-focus), $3 \mathrm{AU}$ (intermediate, mild blur) and $4.7 \mathrm{AU}$ (large, severe blur). Alexa Fluor 488 was excitated with a laser of wavelength $488 \mathrm{~nm}$ and Alexa Fluor 568 with $561 \mathrm{~nm}$, respectively.

\section{Input files from microscopy images}

To ensure accurate analysis of the desired cell, we edited the IF images with multiple cells in the field of view by outlining the cell of interest, noting the background value and filling the area outside the cell with the background. This edited image was taken as the input for the FASensor and Filament Sensor modules. In cases where simply cropping the image could isolate the cell of interest, we did so.

\section{Manual annotation by human expert}

Images from all the conditions were completely manually marked for FAs by a human expert (17 images for in-focus set, 17 images for mild blur set, 19 images for severe blur set). FAs were marked using the freehand selection tool in Fiji [20]. The binary mask of marked adhesions were used as input in evaluation against the software's output.

\section{Bulk dataset evaluation analysis}

To avoid detecting noise and artifacts, we set the lower limit of adhesion detection in the software to 10 pixels which corresponds to $0.144 \mathrm{\mu m}^{2}$ and upper limit at $1000 \mathrm{px}$ which corresponds to $14.4 \mathrm{\mu m}^{2}$. The unoptimized routine across the imaging sets is as follows- For In-Focus dataset, Gauss filter (Sigma-1) and Laplace filter (1, 4 neighbor) with Intermodes thresholding at 55 was used. For Mild-Blur dataset, Gauss filter (Sigma-1) and Laplace filter (1.5, 8 neighbor) with Intermodes thresholding at 80 was used. For Severe-Blur dataset, Gauss filter (Sigma-1) and Laplace filter (3, 8 neighbor) with Intermodes thresholding at 80 was used. Further optimization and customization was done according to user discretion. Closing and Fill holes function was not used for bulk analysis adhesion detection. We used 1 percent minimum matching pixels for object matching in evaluation. Thicken lines function was not used in evaluation. Areas of found and missed adhesions were derived from the pixels column for the user's mask in the result table. Areas of false positive adhesions were derived from the pixels column of the software output in the result table. Pixel values from software results were converted to corresponding micron values using the scale of input image and plotted on graphs. Ordinary one-way ANOVA followed by Dunnett's multiple comparisons test or Tukey's multiple comparisons test was performed using GraphPad Prism (Ver 9.0.0 for Windows, GraphPad Software, San Diego, California USA, www.graphpad.com). For the in-focus set FA-filament correlation analysis, we used the optimization method where each cell had a custom optimal pre-processing filter setting in FASensor. For Filament Sensor, the default settings were used for all cells. For verification, we chose ellipse and a neighborhood of 1 .

\section{Batch threshold determination with ThresholdFinder}

The ThresholdFinder application is an additional software tool that we provide alongside the FAFCK. From a small amount of user-annotated masks, it determines best applicable thresholding algorithm and setting in the FAFCK software. From the input of original images and binary annotations, the software uses the mask to determine desired regions of the image and feeds this into all thresholding algorithms. To determine found, false positive and false negative rates, the images are processed 
bioRxiv preprint doi: https://doi.org/10.1101/2021.04.14.439781; this version posted April 14, 2021. The copyright holder for this preprint (which was not certified by peer review) is the author/funder, who has granted bioRxiv a license to display the preprint in perpetuity. It is made available under aCC-BY 4.0 International license.

whole with the selected algorithm. The value that the respective algorithm would chose without mask input is given, too. 


\section{References}

1. Pellegrin, S.; Mellor, H. Actin stress fibres. Journal of cell science 2007, 120(20):3491-3499

2. Zemel, A.; Rehfeldt, F.; Brown, A.E.X.; Discher, D.E.; Safran, S.A. Optimal matrix rigidity for stress-fibre polarization in stem cells. Nature Physics 2010;6(6): $468-473$

3. Zemel, A.; Rehfeldt, F.; Brown, A.E.X.; Discher, D.E.; Safran, S.A. Cell shape, spreading symmetry, and the polarization of stress-fibers in cells. Journal of Physics: Condensed Matter 2010; 22(19): 194110

4. Eltzner, B.; Wollnik, C.; Gottschlich, C.; Huckemann, S.; Rehfeldt, F. The Filament Sensor for Near Real-Time Detection of Cytoskeletal Fiber Structures. PLOS ONE 10 2015; e0126346.

5. Parsons, J.T.; Horwitz, A.R.; Schwartz, M.A. Cell adhesion: integrating cytoskeletal dynamics and cellular tension. Nature reviews Molecular cell biology 2010, 11(9):633-643

6. Ciobanasu, C.; Faivre, B.; Le Clainche, C. Actin dynamics associated with focal adhesions. International journal of cell biology 2012, 10.1155/2012/941292

7. Soine, J.R.D.; Brand, C.A.; Stricker, J.; Oakes, P.W.; Gardel, M.L.; Schwarz, U.S. Model-based Traction Force Microscopy Reveals Differential Tension in Cellular Actin Bundles. PLOS Computational Biology 2015; 10.1371/journal.pcbi.1004076.

8. Vallenius, T. Actin stress fibre subtypes in mesenchymal-migrating cells. Open biology 2013, 3(6):130001

9. Guo, F.; Debidda, M.; Yang, L.; Williams, D. A.; Zheng, Y. Genetic deletion of Rac1 GTPase reveals its critical role in actin stress fiber formation and focal adhesion complex assembly. Journal of Biological Chemistry 2006; 281(27): $18652-18659$

10. Kanellos, G.; Zhou, J.; Patel, H.; Ridgway, R.A.; Huels, D.; Gurniak, C.B.; Sandilands, E.; Carragher, N.O.; Sansom, O.J.; Witke, W.; Valerie G. B.; Margaret C. F. ADF and Cofilin1 control actin stress fibers, nuclear integrity, and cell survival. Cell reports 2015; 13(9): 1949-1964

11. Bach, C.T.; Creed, S.; Zhong, J.; Mahmassani, M.; Schevzov, G.; Stehn, J.; Cowell, L.N.; Naumanen, P.; Lappalainen, P.; Gunning, P.W.; O'Neill, G.M. Tropomyosin isoform expression regulates the transition of adhesions to determine cell speed and direction. Molecular and cellular biology 2009; 29(6): 1506-1514

12. Feng, Y.; Ngu, H.; Alford, S.K.; Ward, M.; Yin, F.; Longmore, G.D $\alpha$-Actinin1 and 4 tyrosine phosphorylation is critical for stress fiber establishment, maintenance and focal adhesion maturation. Experimental cell research 2013; 319(8): 1124-1135

13. Kovac, B.; Teo, J.L.; Mäkelä, T.P.; Vallenius, T. Assembly of non-contractile dorsal stress fibers requires $\alpha$-actinin- 1 and Rac1 in migrating and spreading cells. Journal of cell science 2013; 126(1): 263-273

14. Berginski, M.E.; Gomez, S.M. The Focal Adhesion Analysis Server: a web tool for analyzing focal adhesion dynamics. F1000Research 2013, 10.12688/f1000research.2-68.v1. 
15. Broussard, J.A.; Diggins, N.L.; Hummel, S.; Georgescu, W.; Quaranta, V.; Webb, D.J. Automated Analysis of Cell-Matrix Adhesions in 2D and 3D Environments. Scientific Reports 2015, 10.1038/srep08124.

16. Buskermolen, A.B.C.; Kurniawan, N.A.; Bouten, C.V.C. An automated quantitative analysis of cell, nucleus and focal adhesion morphology. PLOS ONE 2018, 10.1371/journal.pone.0195201.

17. Nowak, J.; Gennermann, K.; Persson, S.; Nikoloski, Z. CytoSeg 2.0: automated extraction of actin filaments. Bioinformatics 2020, 36(9); 10.1093/bioinformatics/btaa035.

18. Rogge, H.; Artelt, N.; Endlich, N.; Endlich, K. Automated segmentation and quantification of actin stress fibres undergoing experimentally induced changes. Journal of Microscopy 2017, 268(2); 10.1111/jmi.12593.

19. Zhang, Z.; Xia, S.; Kanchanawong, P. An integrated enhancement and reconstruction strategy for the quantitative extraction of actin stress fibers from fluorescence micrographs. BMC Bioinformatics 2017, 18(1); 10.1186/s12859-017-1684-y.

20. Schindelin, J.; Arganda-Carreras, I.; Frise, E.; Kaynig, V.; Longair, M.; Pietzsch, T.; Preibisch, S.; Rueden, C.; Saalfeld, S.; Schmid, B.; Tinevez, J.Y.; White, D.J.; Hartenstein, V.; Eliceiri, K.; Tomancak, P.; Cardona, A. Fiji: an open-source platform for biological-image analysis. Nature Methods 2012, 9(7); 10.1038/nmeth.2019.

21. Schneider, C.A.; Rasband, W.S.; Eliceiri, K.W. NIH Image to ImageJ: 25 years of image analysis. Nature methods 2012; 9(7): 671-675, PMID 22930834. 
A.

Actin

B.

Paxillin

2

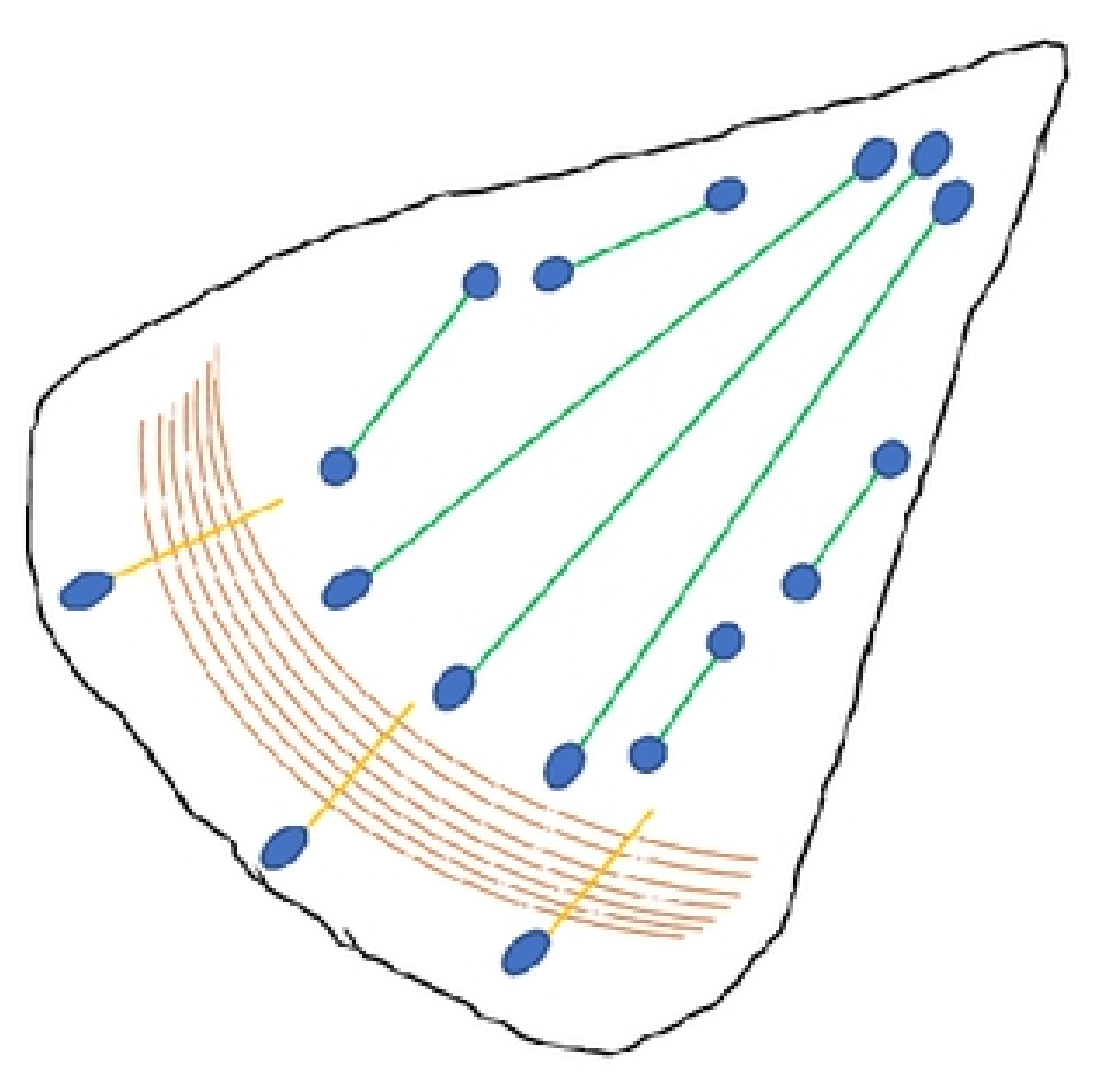

Merge D.

Focal Adhesion Dorsal Stress Fiber | Transverse Arc | Ventral Stress Fiber

C.

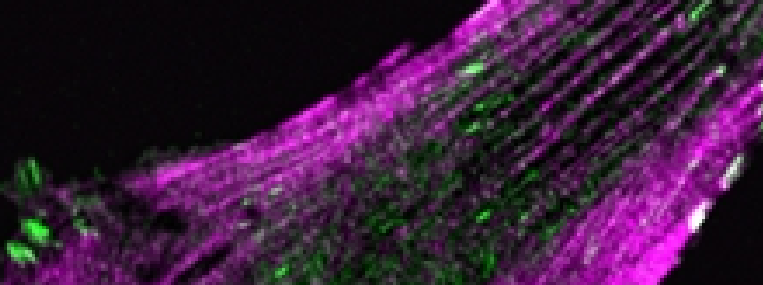

Q. $2,0,0.62$

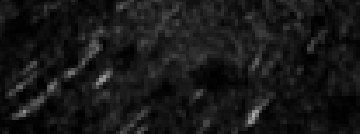




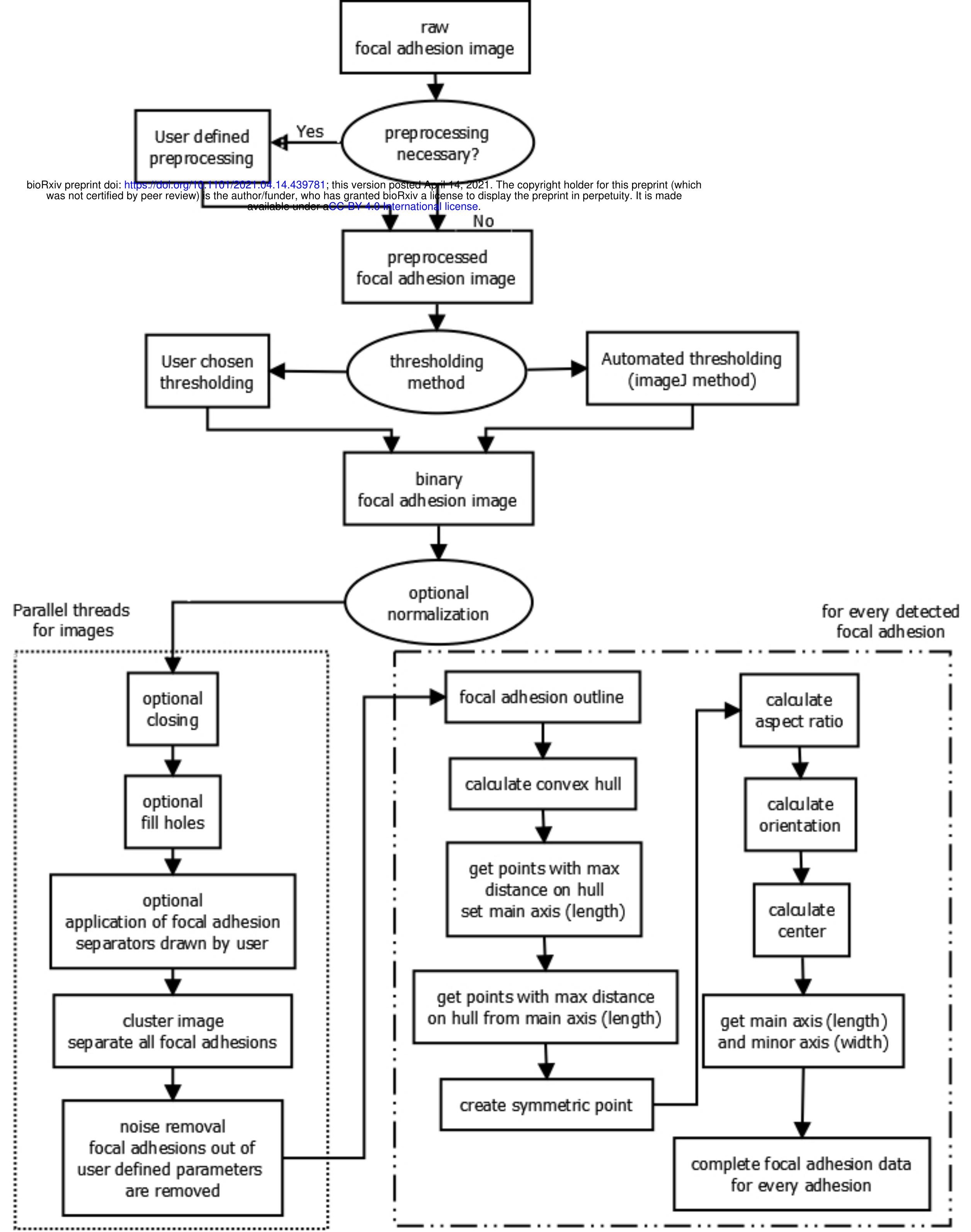


A.

Paxillin

B.

FAsensor input

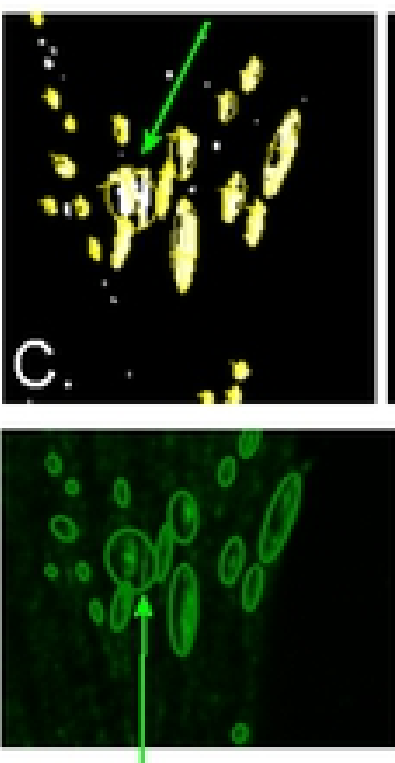

One object

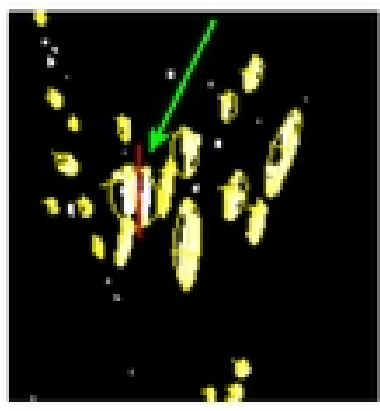

Adhesion Splitting
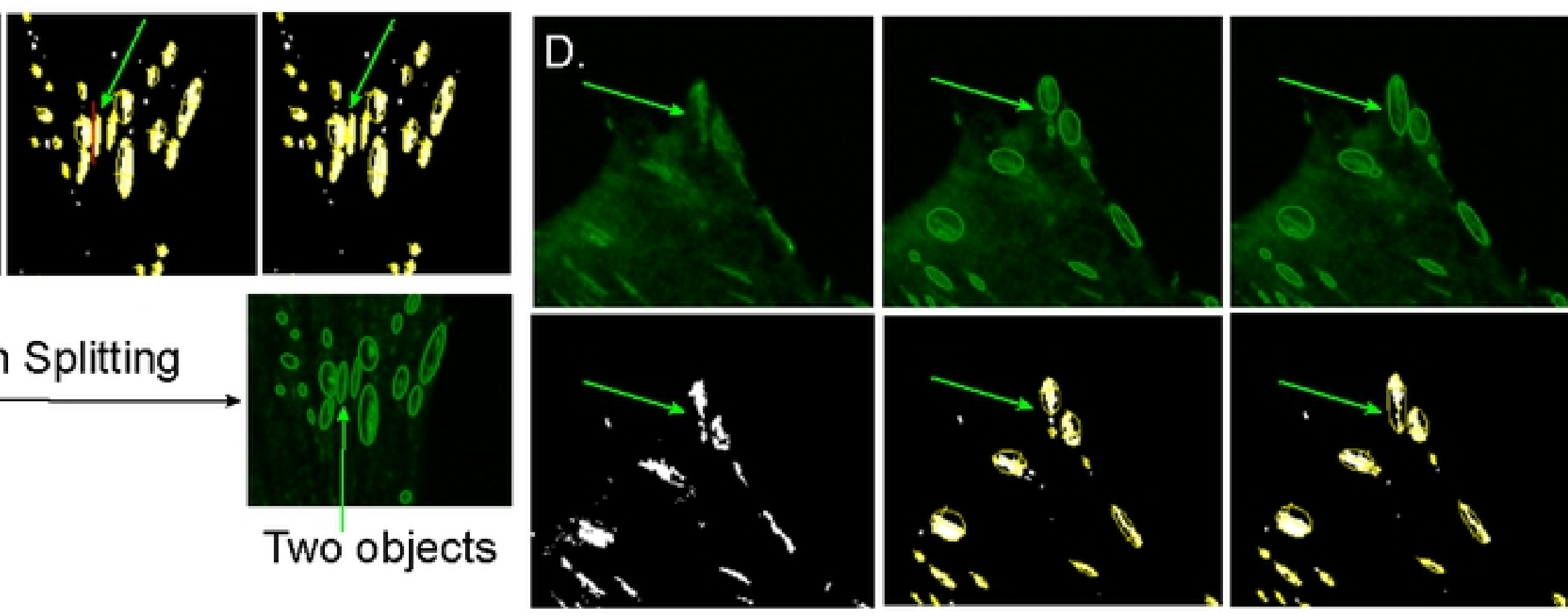

Pre-Detection

With Algorithm 
A.In-focus set B. Mild-blur set

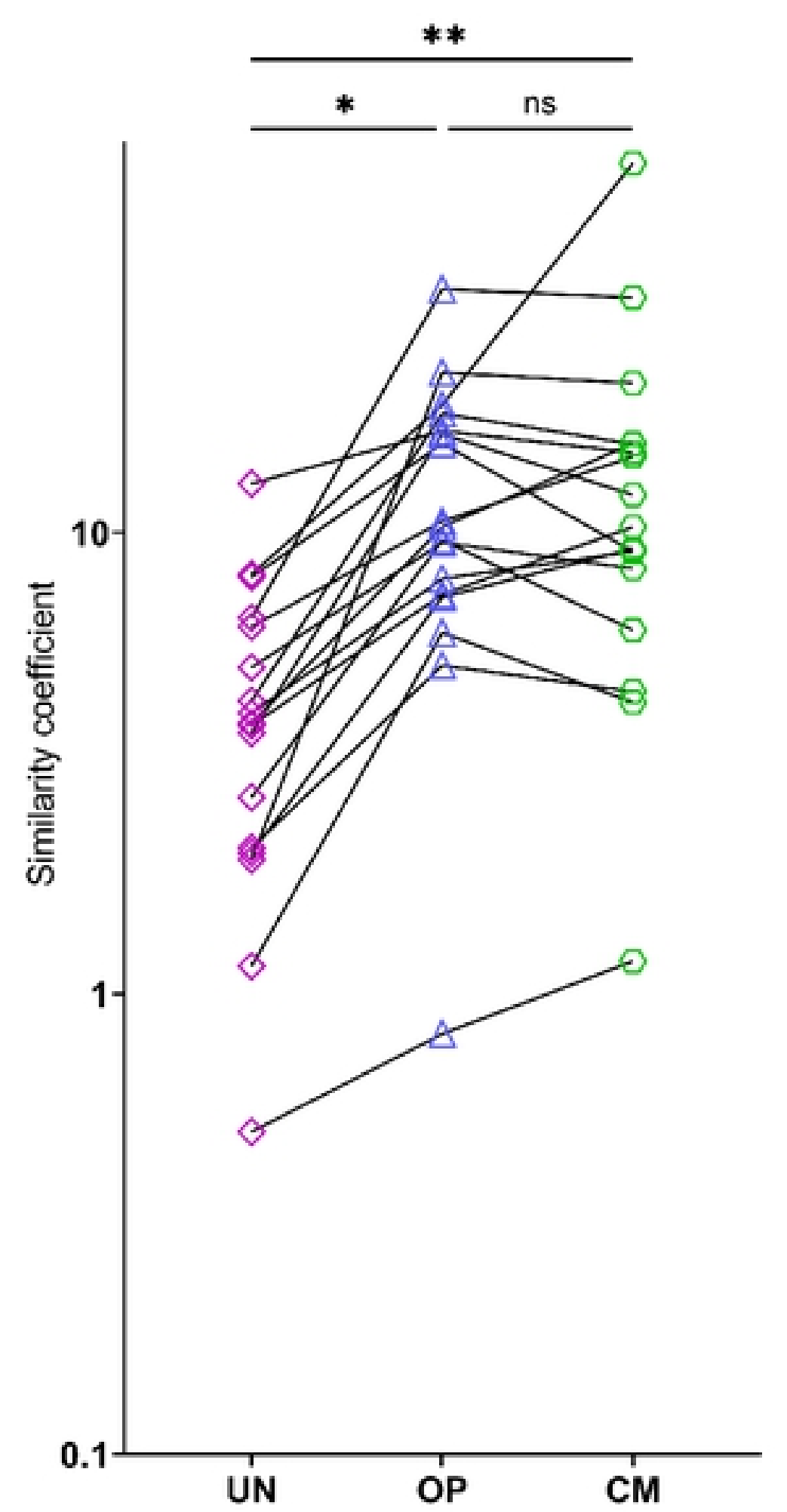

C. Severe-blur set

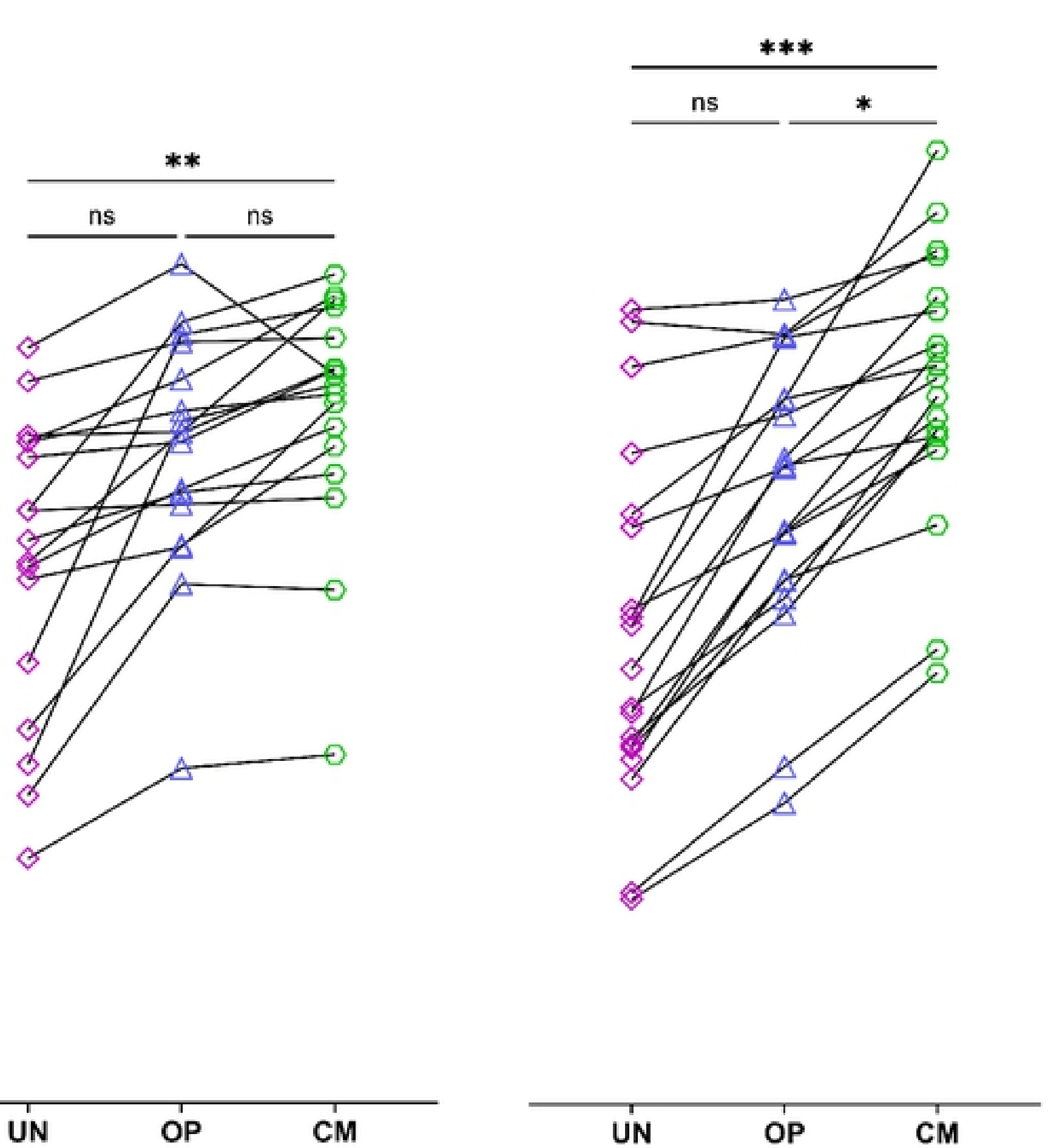


A. In-focus set

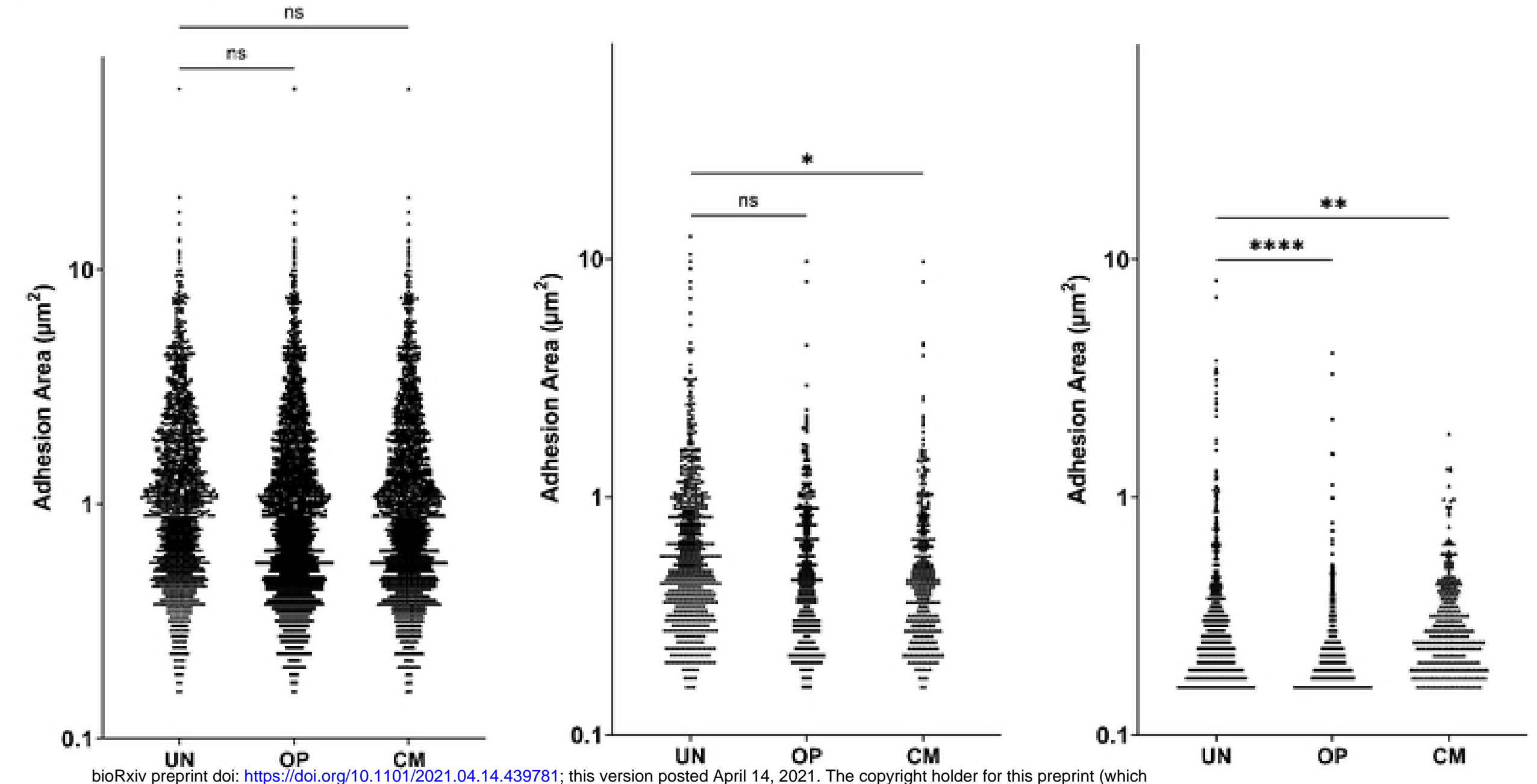

B. Mild-blurs not certified by peer review) is the authorffunder, who has granted bioRxiv a license to display the preprint in perpetuity. It is made
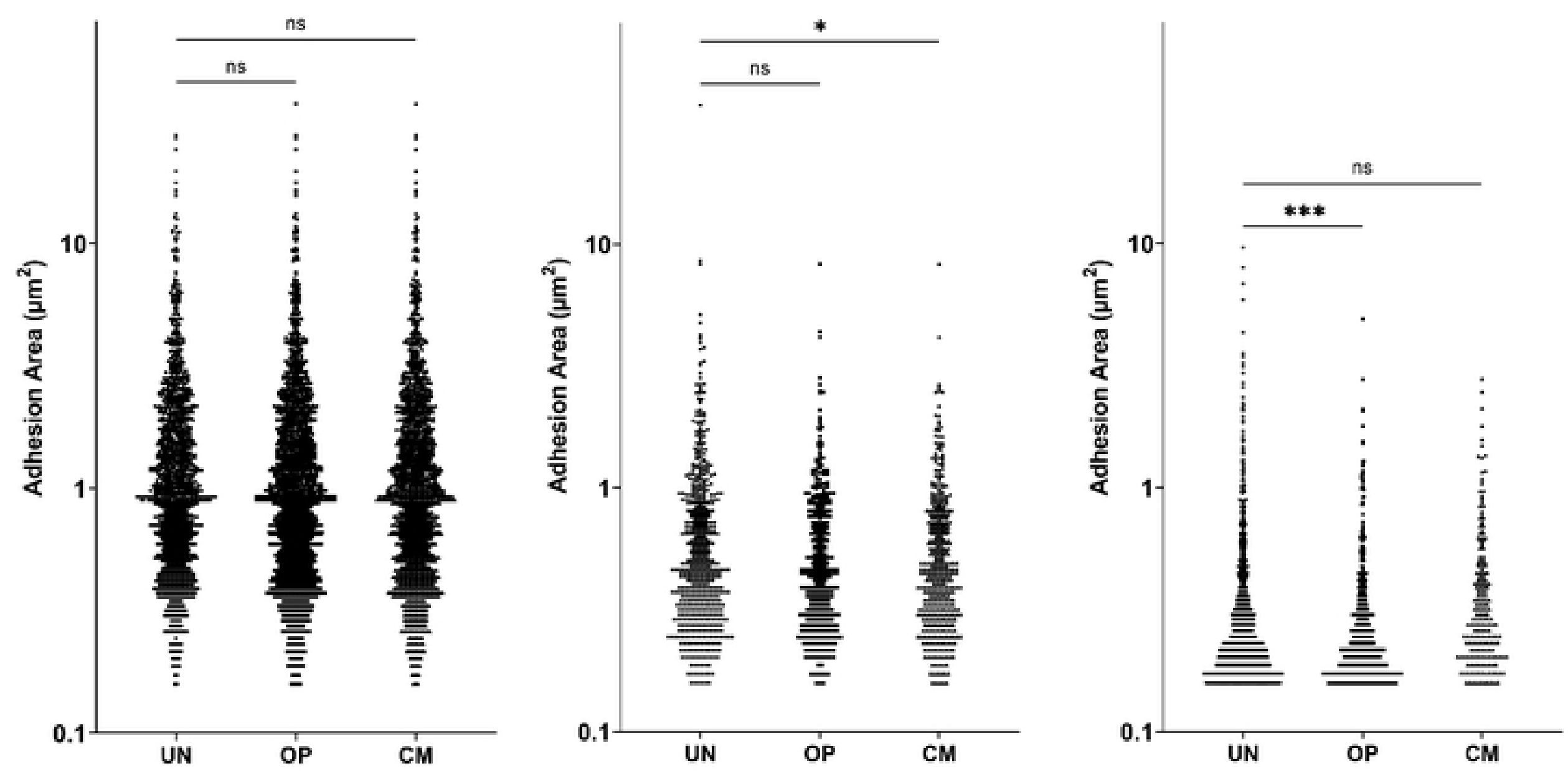

C. Severe-blur set
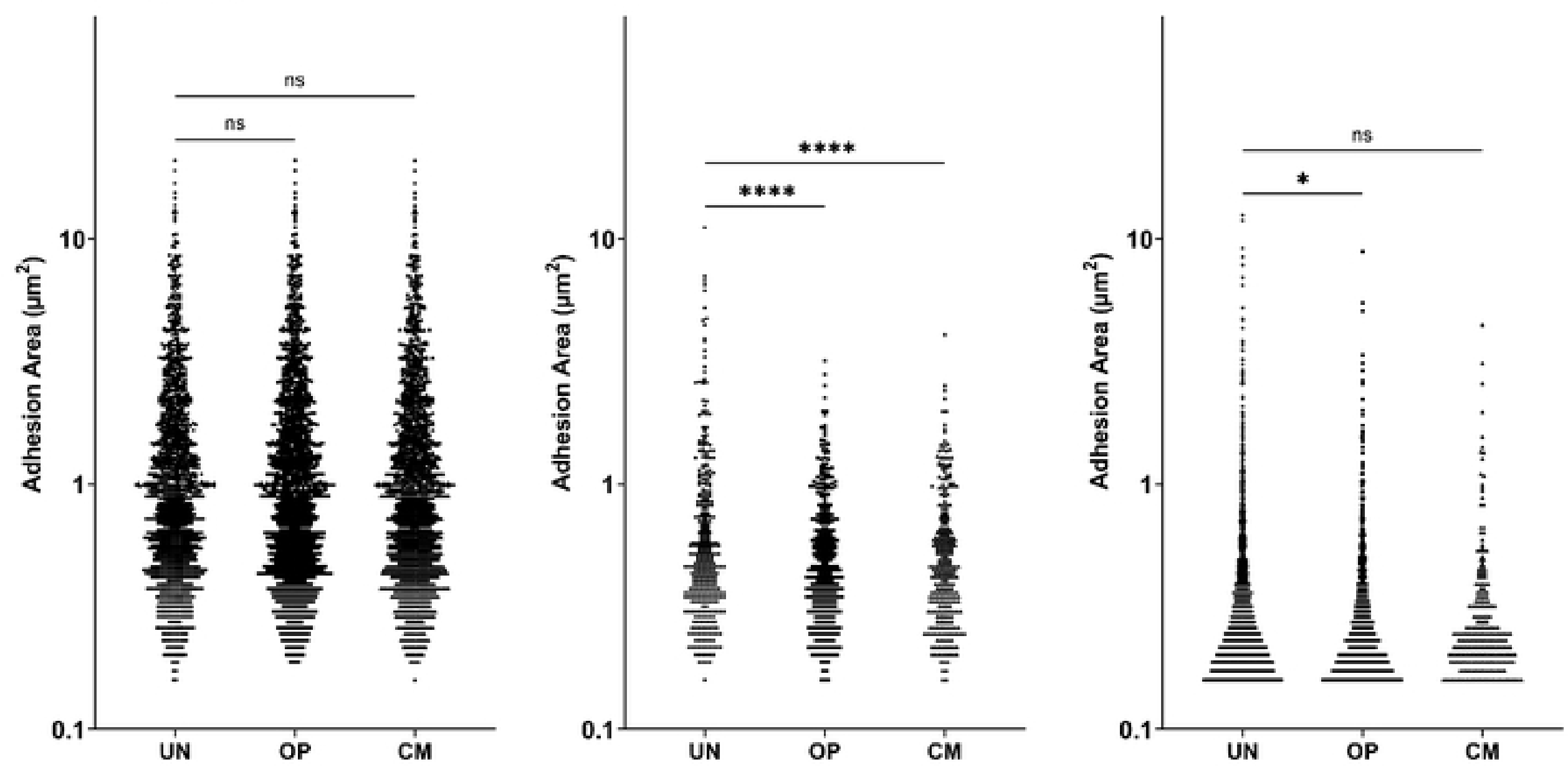
filter data

remove sets where only one object list is found

bioRxiv preprint doi: https:// doi.org/10.1101/2021.04.14.439781; th was not certified by pe review) is the author/funder, who has available under aCC

map data sets per image name

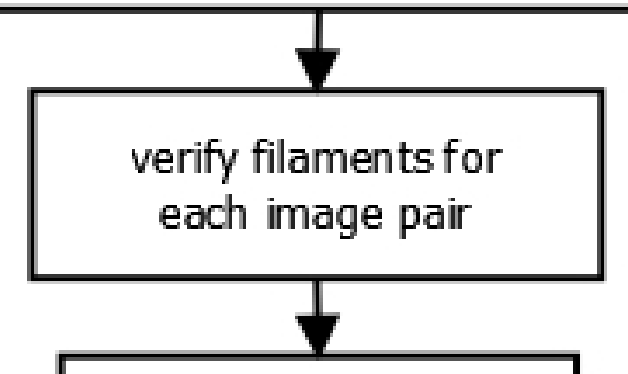

reset verified markers

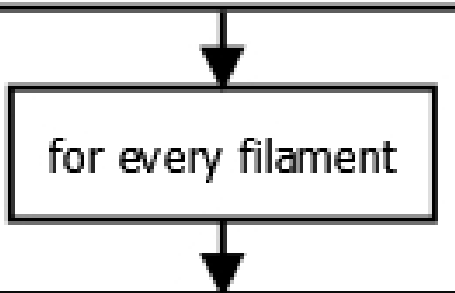

for every focal adhesion (sorted by main axis length)

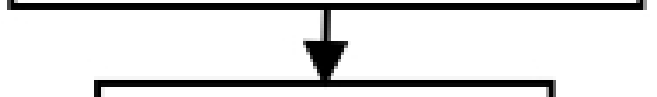

get user chosen verification method

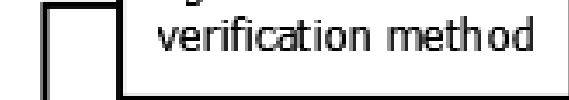

convex hull shape

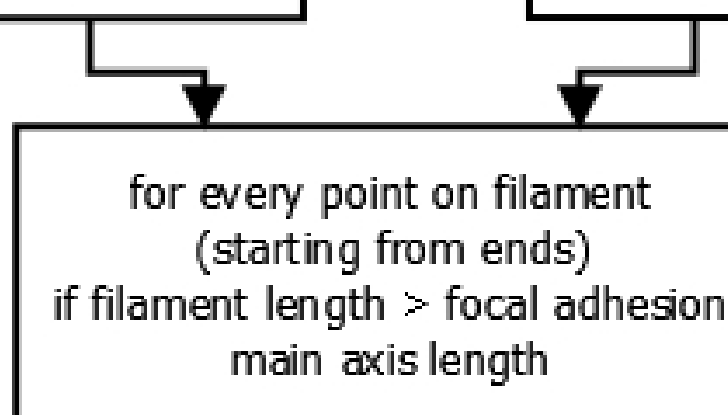

elliptic shape
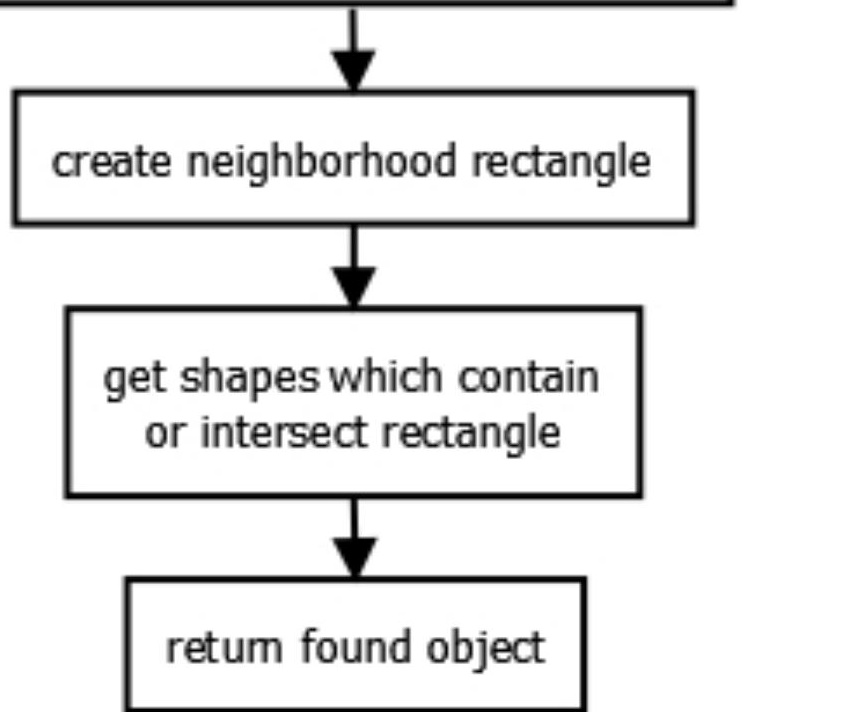
